\title{
Rapid evolution of complete dosage compensation in Poecilia
}

2

\author{
David C.H. Metzger ${ }^{1 *}$, Benjamin A. Sandkam ${ }^{1}$, Iulia Darolti ${ }^{1}$, Judith E. Mank ${ }^{1,2}$
}

4

$5 \quad{ }^{1}$ Department of Zoology and Biodiversity Research Centre, University of British Columbia, Canada

$6 \quad{ }^{2}$ Department of Genetics, Evolution and Environment, University College London, U.K.

7

8

9 * Author for Correspondence: David C.H. Metzger, Department of Zoology and Biodiversity Research

10 Centre, University of British Columbia, Canada, dmetzger@zoology.ubc.ca

11

12

13

14

15

16

17

18

19

20

21

22

23

24

25 


\section{ABSTRACT}

27 Dosage compensation balances gene expression between the sexes in systems with diverged

28 heterogametic sex chromosomes. Theory predicts that dosage compensation should rapidly

29 evolve in parallel with the divergence of sex chromosomes to prevent the deleterious effects of

30 dosage imbalances that occur as a result of sex chromosome divergence. Examples of complete

31 dosage compensation, where gene expression of the entire sex chromosome is compensated, are

32 rare and have only been found in relatively ancient sex chromosome systems. Consequently,

33 very little is known about the evolutionary dynamics of complete dosage compensation systems.

34 We recently found the first example of complete dosage compensation in a fish, Poecilia picta.

35 We also found that the Y chromosome degraded substantially in the common ancestor of $P$. picta

36 and their close relative Poecilia parae. In this study we find that $P$. parae also have complete

37 dosage compensation, thus complete dosage compensation likely evolved in the short ( 3.7 my)

38 interval after the split of the ancestor of these two species from $P$. reticulata, but before they

39 diverged from each other. These data suggest that novel dosage compensation mechanisms can

40 evolve rapidly, thus supporting the longstanding theoretical prediction that such mechanisms

41 arise in parallel with rapidly diverging sex chromosomes.

43 Keywords: RNA-seq, sex chromosome, Y degeneration, Poecilia parae 


\section{SIGNIFICANCE STATEMENT}

50 In species with XY sex chromosomes, females (XX) have as many copies of X-linked genes compared to

51 males (XY), leading to unbalanced expression between the sexes. Theory predicts that dosage

52 compensation mechanisms should evolve rapidly as $\mathrm{X}$ and $\mathrm{Y}$ chromosomes diverge, but examples of

53 complete dosage compensation in recently diverged sex chromosomes are scarce, making this theory

54 difficult to test. Across Poeciliid species the X and Y chromosomes have recently diversified. Here we

55 find complete dosage compensation evolved rapidly as the $\mathrm{X}$ and $\mathrm{Y}$ diverged in the common ancestor of

56 Poecilia parae and P. picta, supporting that novel dosage compensation mechanisms can evolve rapidly

57 in tandem with diverging sex chromosomes. These data confirm longstanding theoretical predictions of

58 sex chromosome evolution.

\section{INTRODUCTION}

61 In organisms with heterogametic sex determination, the Y chromosome diverges from the

$62 \mathrm{X}$ when recombination between them is suppressed (Furman et al. 2020). The same process

63 holds for the $\mathrm{Z}$ and $\mathrm{W}$ chromosomes, but we focus here on male heterogametic systems.

64 Degradation of the $\mathrm{Y}$ chromosome can lead to pseudogenization and gene loss resulting in

65 females (XX) having twice as many copies of genes on the sex chromosome compared to males

66 (XY). Because genes are normally expressed equally from both copies of a chromosome, males

67 would only have half the expression of X-linked loci (Ohno 1967; Gu \& Walters 2017), leading

68 to a dosage imbalance with expression of genes on the autosomes. To resolve this issue, many

69 organisms have evolved mechanisms to equalize expression levels of these sex chromosome

70 genes, known as dosage compensation (Ohno 1967). Dosage compensation mechanisms are

71 thought to evolve rapidly in parallel with Y degradation (Ohno 1967), however, the majority of 
72 sex chromosomes with dosage compensation are relatively old making it difficult to determine if

73 dosage compensation can evolve in rapidly diverging sex chromosome systems.

74 Dosage compensation can either act by modifying expression on a gene-by-gene basis

75 (incomplete dosage compensation) or by modifying expression along the entire chromosome

76 (complete dosage compensation). Complete dosage compensation is predicted to arise for sex

77 chromosomes that are rapidly diverging and experiencing extensive gene loss or

78 pseudogenization, and has been more commonly found in male-heterogametic systems (XY)

79 (Mullon et al. 2015; Wilson Sayres \& Makova 2011). The most well characterized example for

80 the rapid evolution of complete dosage compensation is in Drosophila where complete dosage

81 compensation followed the emergence and divergence of a new XY sex chromosome system

82 (Marín et al. 1996). The emergence of dosage compensation on neo-sex chromosomes in

83 Drosophila is the result of evolution coopting extant dosage compensation mechanisms that

84 predate the origin of the Drosophila genus (Marín et al. 1996). While dosage compensation can

85 clearly evolve rapidly, it is unknown if complete dosage compensation can evolve rapidly when

86 it is not present in close relatives.

87 Fish exhibit a high rate of sex chromosome turnover, and although there are some species

88 with incomplete dosage compensation (eg. sticklebacks, flatfish, and rainbow trout) (White et al.

89 2015; Shao et al. 2014; Hale et al. 2018) complete dosage compensation appears to be rare. We

90 recently identified the first example of complete dosage compensation in a fish; Poecila picta. P.

91 picta is a close relative to the guppy (Poecila reticulata) (Darolti et al. 2019) that shares the same

92 XY system that originated 18.48-26.08 Mya (Darolti et al. 2019; Rabosky et al. 2018). In $P$.

93 reticulata, the $\mathrm{X}$ and $\mathrm{Y}$ have remained largely homomorphic, with little evidence of gene loss on

94 the Y, and no need for dosage compensation (Darolti et al. 2019). However, since their split 
95 18.4 Mya (Rabosky et al. 2018) the P. picta $\mathrm{Y}$ has diverged substantially from the $\mathrm{X}$ across

96 nearly the entire chromosome and evolved complete dosage compensation (Darolti et al. 2019).

Here we take a comparative approach to narrow the timing of the evolution of complete

98 dosage compensation by testing for dosage compensation in P. parae a sister taxon to P. picta.

99 We recently characterized the sex chromosomes of $P$. parae, including five discrete $\mathrm{Y}$

100 haplotypes that control the five male morphs of this species (Sandkam et al. 2020). Importantly

101 we found XY divergence across all five $P$. parae Ys was the same as XY divergence in $P$. picta,

102 indicating the $\mathrm{Y}$ diverged from the $\mathrm{X}$ in the $\sim 3.7$ my interval spanning the split of the $P$. picta-

$103 P$. parae from the common ancestor with $P$. reticulata $\sim 18.4$ mya, and prior to the split of $P$.

104 picta and $P$. parae from each other $\sim 14.7$ mya (Rabosky et al. 2018). Therefore, if P. parae also

105 has complete dosage compensation, then dosage compensation evolved rapidly after XY

106 chromosome divergence over a period of less than 3.7 million years (Figure 1).

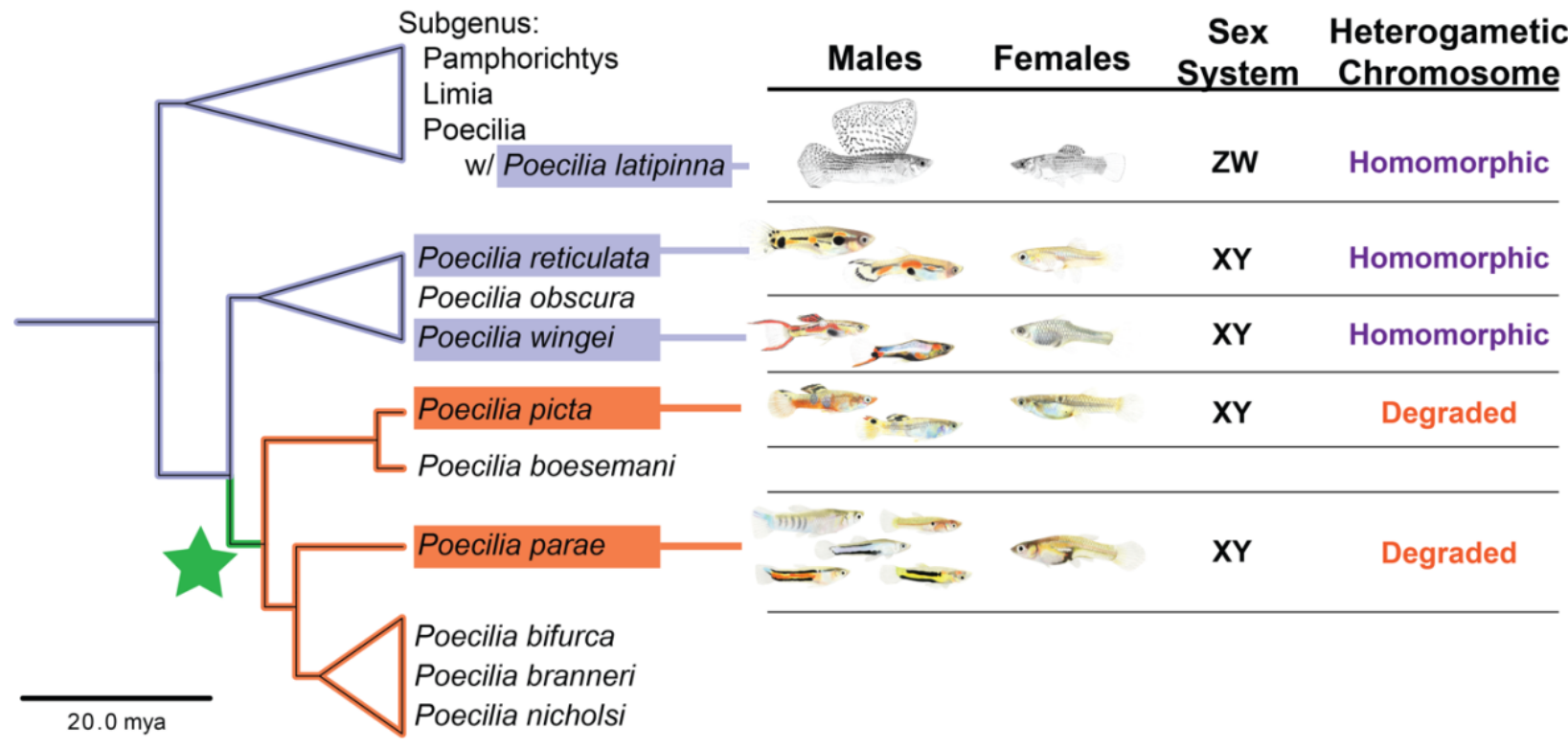


Figure 1. A phylogeny of Poecilia species depicting the timeframe in which dosage compensation systems observed in $P$. picta and $P$. parae evolved over the $\sim 3.7$ million year interval (denoted in green)

112 after the common ancestor to $P$. picta-P. parae split from $P$. reticulata-P.wingei $(\sim 18.4$ mya) and prior to

113 the divergence of $P$. picta and $P$. parae from each other ( 14.7 mya). The branch where sex chromosome

114 divergence and dosage compensation evolved is indicated in green. Orange branches indicate the clade

115 containing species where $\mathrm{X}$ and $\mathrm{Y}$ are substantially diverged and have complete dosage compensation $(P$.

116 picta- Darolti et al 2019, P. parae- this study). Blue indicates species for which dosage compensation has

117 been explicitly tested but found to be entirely lacking (Darolti et al 2019). Green star denotes the branch

118 on which complete dosage compensation likely evolved. The phylogeny and divergence times are taken

119 from The Fish Tree of Life (Rabosky et al. 2018).

\section{RESULTS}

\section{Characterization of dosage compensation in P. parae} with the five different male morphs (immaculata, yellow melanzona, blue melanzona, red

127 melanzona, and parae morphs). Importantly, these five $P$. parae $\mathrm{Y}$ haplotypes emerged after X-Y

128 recombination was halted before the split between $P$. picta and P. parae, 18.4 Mya (Sandkam

129 et al. 2020; Lindholm et al. 2004). Therefore, if complete dosage compensation evolved in the

130 common ancestor of $P$. picta and $P$. parae, we would expect to see dosage compensation in all

131 male morphs as well. To assess this, we tested for differences in expression from the $\mathrm{X}$ and $\mathrm{Y}$

132 chromosome in three of the five male morphs (yellow melanzona, blue melanzona, and parae,

133 hereafter referred to as yellow, blue, and parae males). It is worth noting that all five Y 
134 haplotypes show similar patterns of divergence from the X (Sandkam et al. 2020), and so the

135 three morphs we assessed here are indicative of the species as a whole.

136 For genes that are equally expressed from both sex chromosomes we expect to see a

137 similar proportion of transcripts expressed from each sex chromosome. To test this, we first

138 identified heterozygous transcripts. We found that $17 \%$ of the 38,986 autosomal transcripts

139 exhibit heterozygous expression in both males and females, and a similar proportion (12\%) of

140 the1,349 transcripts from the sex chromosome are heterozygous in females. In contrast, only $1 \%$

141 of sex chromosome transcripts are heterozygous in $P$. parae males. These data suggest that

142 widespread gene loss has occurred as a result of Y chromosome divergence in males.

143 We then compared the major allele ratios for heterozygous transcripts. Autosomal genes

144 are equally expressed from both chromosomes in both sexes, and in X-linked genes in P. parae

145 females (Figure 2A/B). However, in males we found significant allele specific expression (ASE)

146 for sex-linked genes, consistent with the notion that for sex-linked genes that remain

147 heterozygous in males, gene activity has been reduced from the Y paralog and expression is

148 primarily produced from the X. This pattern was convergent across each male morph (Figure

149 2A). 

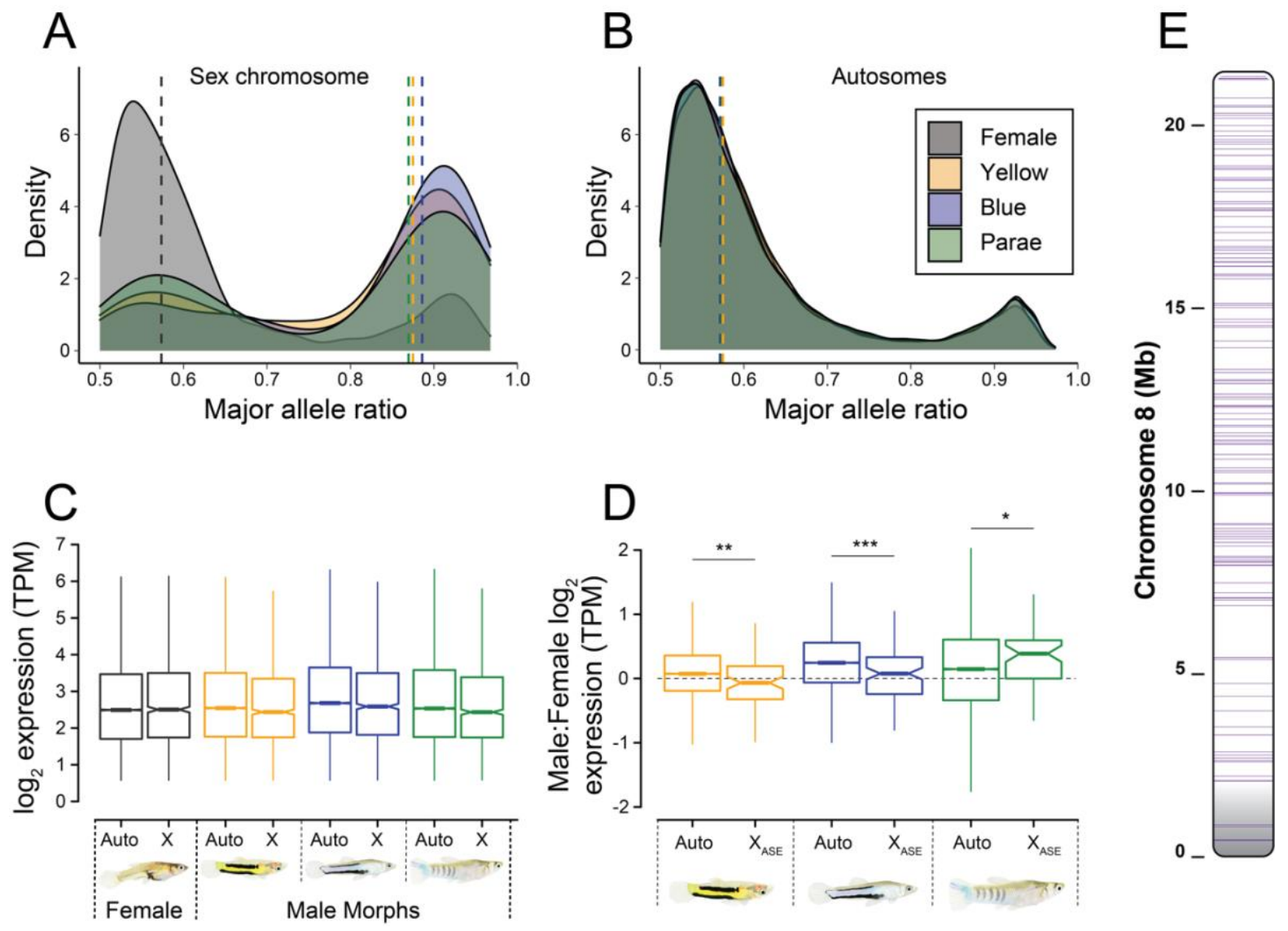

Figure 2. Patterns of allele specific expression (ASE) on the sex chromosome (A) and the autosomes (B)

153 for females and each of the three male morphs of P. parae indicate loss of expression for genes encoded

154 on the Y. ASE ratio of 0.5 indicates equal expression from both copies of a chromosome, while shifts

155 toward 1 indicate expression predominantly comes from just one copy. Vertical dashed lines are median

156 major allele ratio values. (C) Despite loss of expression from the Y, expression levels ( $\log 2$ transcripts per

157 million (TPM)) of sex chromosome genes do not differ from the autosomes for any of the male morphs.

158 (D) Male:female expression ratios for genes that exhibit allele specific expression are not different from

159 male:female expression ratios of autosomal genes, demonstrating that a loss of expression from the Y

160 chromosome in males does not result in reduced expression. The horizontal dashed line represents equal

161 expression between males and females. Colours are consistent in all panels and denote sex and/or male

162 morph. Grey = female, Yellow = yellow male morph, blue = blue male morph, green = parae male morph. 
(E) Distribution of genes with allele specific expression (ASE) on the male X chromosome (chromosome

8). ASE genes are evenly distributed along the entire chromosome, confirming complete dosage compensation for genes on the sex chromosome. Gene locations are demarcated by purple lines. The pseudo autosomal region (PAR) is in grey.

172 (Wilcoxon rank sum yellow $\mathrm{p}$-value $=0.9703$, blue $\mathrm{p}$-value $=0.4965$, parae $\mathrm{p}$-value $=0.292$ ), or

173 between genes on the $\mathrm{X}$ chromosome or autosomes in females (Wilcoxon rank sum $\mathrm{p}$-value $=$

174 0.8336). Together this indicates complete dosage compensation arose before the morphs

175 diverged and likely in the common ancestor of $P$. picta and $P$. parae (Figure 2C).

177 linked genes with an ASE pattern in the yellow $(\mathrm{p}$-value $=0.001)$ and blue $(\mathrm{p}$-value $=0.0001)$

178 male morphs compared to genes on the autosomes which is consistent with the expression

179 pattern in $P$. picta (Darolti et al., 2019). In contrast, the male:female expression ratio for sex

180 chromosome genes with ASE in the parae male morph were significantly higher compared to the

181 male:female ratio of autosomal genes $(p$-value $=0.05)($ Figure $2 \mathrm{D})$. These data indicate that the

182 efficiency of the dosage compensation in P. parae is similar to P. picta and that there may be

183 residual Y-linked expression of these genes or that dosage compensation results in a moderate

184 overexpression of some X-linked genes in males. 
187 is silenced in females. Although this balances sex chromosome gene expression between males

188 and females, it does not address expression differences between X-linked and autosomes genes.

189 In fact, $\mathrm{X}$ inactivation in females means that both sexes on average express $\mathrm{X}$-linked genes less

190 than the autosomal average, and only dosage sensitive genes on the $\mathrm{X}$ are upregulated in both

191 sexes to counter this (Pessia et al. 2012). Alternatively, in Drosophila (Marín et al. 2000), and

192 Anolis (Marin et al. 2017), dosage compensation is achieved by doubling the expression of genes

193 on the $\mathrm{X}$ chromosome in males.

194 We found that expression of genes on the X chromosome is not different from expression

195 of genes on the autosomes in females, or any of the male morphs, and that the major allele ratio

196 for X-linked genes in females is close to 0.5 indicating roughly equal expression from both

197 copies of the X. Furthermore, ASE genes in males are distributed along the entire X chromosome

198 providing further support for dosage compensation of the entire chromosome (Figure 2E). Taken

199 together, these data suggest that complete dosage compensation in P. parae is more similar to

200 dosage compensation in Drosophila and Anolis where genes on the $\mathrm{X}$ are hyper expressed in

201 males. This provides an excellent avenue to explore the mechanisms controlling expression

202 across entire chromosomes.

\section{DISCUSSION}

205 Rapid evolution of dosage compensation

206 Although theory suggests complete dosage compensation should evolve rapidly in

207 tandem with Y degradation (Ohno 1967), gene expression studies in non-model systems with

208 heteromorphic sex chromosomes have demonstrated that complete dosage compensation is

209 actually quite rare and is not a guaranteed outcome of sex chromosome evolution (Mank et al. 
210 2011). These studies show that there are many alternatives to evolving complete dosage

211 compensation, and that complete dosage compensation is an exceptional outcome of sex

212 chromosome evolution. Until the recent characterization of complete dosage compensation in $P$.

213 picta, complete dosage compensation has been observed in a limited number of lineages, all of

214 which are relatively ancient (Marin et al. 2017; Mullon et al. 2015). The age of these systems

215 makes it difficult to refine estimates for the speed at which complete dosage compensation can

216 arise.

217 Within the family Poeciliidae the subgenus Lebistes is particularly well suited to address

218 this question as it contains several species with characterized sex chromosomes including $P$.

219 reticulata, $P$. wingei, $P$. picta, and $P$. parae (Darolti et al. 2019). There is strong evidence that all

220 Lebistes share the same sex chromosome system which originated 18.48-26.08 Mya (Darolti et

221 al. 2019; Rabosky et al. 2018). Despite sharing the same XY system, the extent of Y degradation

222 differs dramatically, from largely intact in $P$. reticulata and $P$. wingei to highly degraded in $P$.

223 picta and P. parae (Darolti et al. 2019; Sandkam et al. 2020). Without gene loss, there would be

224 no selective pressure to evolve dosage compensation, thus it is not surprising that a dosage

225 compensation was not found in either P. reticulata and $P$. wingei (Darolti et al. 2019), where

226 there is little evidence of decreased gene activity from the Y chromosome.

227 In contrast, the Y chromosomes in P. picta and P. parae exhibit substantial divergence

228 along the entire chromosome (Sandkam et al. 2020; Darolti et al. 2019). Here we present

229 evidence for complete dosage compensation common in multiple morphs of $P$. parae. These data

230 suggest that the dosage compensation system in $P$. parae is shared with $P$. picta and evolved

231 over a period of less than 4 million years in their common ancestor. 
In some systems, the rapid evolution of complete dosage compensation is achieved by recruiting an ancestral or pre-existing dosage compensation mechanism (Marín et al. 1996;

234 Marin et al. 2017). In fishes, complete dosage compensation is rare, which may be the result of

235 frequent sex chromosome turnover and a paucity of heteromorphic sex chromosomes that makes complete dosage compensation unnecessary. As such dosage compensation in fish is frequently

237 accomplished on a gene-by-gene basis and remains overall incomplete (Shao et al. 2014; White 238 et al. 2015; Darolti et al. 2019) with the exception of P. picta (Darolti et al. 2019) and P. parae.

239 Further work elucidating the mechanism of X chromosome dosage compensation P. picta and $P$.

240 parae will provide novel insights in the evolution of dosage compensation mechanisms.

\section{METHODS}

\section{RNA isolation and sequencing}

244 Animals used in this study were collected in Spring 2019 from natural populations in Suriname

245 and brought to the University of British Columbia (Vancouver, BC, Canada) aquatics facility,

246 where they were kept in $20 \mathrm{~L}$ glass aquaria on a $12: 12$ day:night cycle at $26^{\circ} \mathrm{C}$ and $6 \mathrm{ppt}$ salinity

247 (Instant Ocean Sea Salt) and fed Hikari Fancy Guppy pellets and live brine shrimp daily.

248 Individuals were euthanized using a lethal overdose of MS-222 and muscular tail tissue was

249 taken from the anal pore to the base of the pectoral fin. RNA was immediately isolated using

250 RNeasy spin columns with on-column DNase treatment (Qiagen) following the manufacturer's

251 recommended protocol. Library preparation and 100bp paired-end sequencing was performed on

252 an Illumina NovaSeq 6000 at McGill University and the Génome Québec Innovation Centre.

253 Adaptor sequences were removed and reads were quality filtered and trimmed using

254 trimmomatic (v0.36) using a sliding window of 4 bases and a minimum Phred score of 15. Reads 
255 with leading and trailing bases with a Phred score $<3$ were also removed. Sequencing libraries

256 consisted of $\sim 88$ million reads.

Transcript Alignment and Filtering

259 Reads were aligned to a previously published female $P$. parae genome assembly (Sandkam et al.

260 2020) using the two-pass method for STAR align (v2.7.2) (Dobin et al. 2013). Alignments were

261 sorted by coordinate and converted to BAM format using SAMtools (v1.9). To find the full list

262 of non-redundant $P$. parae transcripts we generated GTF files for each individual using StringTie

263 (v1.3.6) then merged all GTF files. To remove non-coding RNA (ncRNA) we first compiled a

264 database of all ncRNAs in reference genomes of close relatives on Ensemble: Poecilia formosa

265 (PoeFor_5.1.2), Oryzias latipes (ASM223467v1), Gasterosteus aculeatus (BROAD S1), and

266 Danio rerio (GRCz11). We then removed all P. parae transcripts that BLASTed to our ncRNA

267 database.

269 Allele Specific Expression

270 To ensure our results are comparable to our previous results in P. picta we followed the same

271 pipeline to identify allele specific expression (Darolti et al. 2019). In short, for each sex and

272 morph we identified SNPs separately using SAMtools mpileup (v1.9) and varscan (v2.4.3) with

273 parameters --min-coverage 2, --min-ave-qual 20, --min-freq-for-hom 0.90, and excluding

274 triallelic SNPs. We then filtered SNPs for a minimum site coverage of 15 to account for

275 sequencing errors, and used a variable coverage filter to account for potential effects of

276 sequencing errors due to variable coverage levels (an error rate of 1 in 100 and a maximum

277 coverage for a given site of 100,000) (Quinn et al. 2014). We then removed SNP clusters of more 
278 than five SNPs in 100bp window to limit potential biases from read assignments to a single

279 reference sequence (Stevenson et al. 2013).

281 Expression Level

282 We extracted read counts using the featureCounts from the subread package (Liao et al. 2014)

283 and the ncRNA filtered GTF file described above. Reads with low expression (less than $10 \%$ of

284 the mean) were removed from the dataset. We then used a Wilcoxon rank sum test to compare

285 expression levels between groups using the wilcox.test () function in $\mathrm{R}(\mathrm{p}<0.05)$.

\section{DATA AVAILABILITY}

288 Illumina fastq read files will be made publicly available on the Genbank sequence read archive 289 upon publication of this manuscript.

\section{ACKNOWLEDGEMENTS}

292 This work was supported by the Canada 150 Research Chair Program and the European

293 Research Council (680951) to JEM. and a Banting Postdoctoral Fellowship to BAS (Natural

294 Science and Engineering Research Council of Canada). We thank Clara Lacy for guppy

295 illustrations and all members of the Mank lab for helpful comments and suggestions. 


\section{REFERENCES}

302

303

304

305

306

307

308

309

310

311

312

313

314

315

316

317

318

319

320

321

322

323

324

325

326

327

328

329

330

331

332

333

334

335

336

337

338

339

Darolti I et al. 2019. Extreme heterogeneity in sex chromosome differentiation and dosage compensation in livebearers. Proceedings of the National Academy of Sciences. 116:1903119036. doi: 10.1073/pnas.1905298116.

Dobin A et al. 2013. STAR: Ultrafast universal RNA-seq aligner. Bioinformatics. 29:15-21. doi: 10.1093/bioinformatics/bts635.

Furman BLS et al. 2020. Sex chromosome evolution: so many exceptions to the rules. Genome Biology and Evolution. 12:750-763. doi: 10.1093/gbe/evaa081.

Gu L, Walters JR. 2017. Evolution of sex chromosome dosage compensation in animals: A beautiful theory, undermined by facts and bedeviled by details. Genome Biology and Evolution. 9:2461-2476. doi: 10.1093/gbe/evx154.

Hale MC, McKinney GJ, Thrower FP, Nichols KM. 2018. Evidence of sex-bias in gene expression in the brain transcriptome of two populations of rainbow trout (Oncorhynchus mykiss) with divergent life histories. PLoS ONE. 13:1-18. doi: 10.1371/journal.pone.0193009.

Liao Y, Smyth GK, Shi W. 2014. FeatureCounts: An efficient general purpose program for assigning sequence reads to genomic features. Bioinformatics. 30:923-930. doi: 10.1093/bioinformatics/btt656.

Lindholm AK, Brooks R, Breden F. 2004. Extreme polymorphism in a Y-linked sexually selected trait. Heredity. 92:156-162. doi: 10.1038/sj.hdy.6800386.

Mank JE, Hosken DJ, Wedell N. 2011. Some inconvenient truths about sex chromosome dosage compensation and the potential role of sexual conflict. Evolution. 65:2133-2144. doi: 10.1111/j.1558-5646.2011.01316.x.

Marín I, Franke A, Bashaw GJ, Baker BS. 1996. The dosage compensation system of Drosophila is co-opted by newly evolved X chromosomes. Nature. 383:160-163. doi: 10.1038/383160a0.

Marín I, Siegal ML, Baker BS. 2000. The evolution of dosage-compensation mechanisms. BioEssays. 22:1106-1114. doi: 10.1002/1521-1878(200012)22:12<1106::AIDBIES8>3.0.CO;2-W.

Marin R et al. 2017. Convergent origination of a Drosophila-like dosage compensation mechanism in a reptile lineage. Genome Research. 27:1974-1987. doi: 10.1101/gr.223727.117. 

compensation under sexual selection differs between $\mathrm{X}$ and $\mathrm{Z}$ chromosomes. Nature Communications. 6:1-10. doi: 10.1038/ncomms8720.

Ohno S. 1967. Sex chromosomes and sex-linked genes. Springer-Verlag: New York. chromosome inactivation evolved as a dosage-compensation mechanism for dosage-sensitive genes on the X chromosome. Proceedings of the National Academy of Sciences of the United States of America. 109:5346-5351. doi: 10.1073/pnas.1116763109.

Quinn A, Juneja P, Jiggins FM. 2014. Estimates of allele-specific expression in Drosophila with

Rabosky DL et al. 2018. An inverse latitudinal gradient in speciation rate for marine fishes. Nature. 559:392-395. doi: 10.1038/s41586-018-0273-1.

Sandkam BA et al. 2020. Extreme Y chromosome polymorphism corresponds to five male reproductive morphs. bioRxiv. doi: 10.1101/2020.08.19.258434.

Shao C et al. 2014. Epigenetic modification and inheritance in sexual reversal of fish. Genome Research. 24:604-615. doi: 10.1101/gr.162172.113. expression derived from RNA-seq data aligned to a single reference genome. BMC Genomics. 14. doi: 10.1186/1471-2164-14-536. during degeneration of the threespine stickleback Y chromosome. Molecular Biology and many species. BioEssays. 33:938-945. doi: 10.1002/bies.201100091. 